ARTICLE

Received 5 Jan 2013 | Accepted 17 May 2013 | Published 25 Jun 2013

DOI: $10.1038 /$ ncomms3030

\title{
Scara1 deficiency impairs clearance of soluble amyloid- $\beta$ by mononuclear phagocytes and accelerates Alzheimer's-like disease progression
}

Dan Frenkel ${ }^{1, \star}$, Kim Wilkinson ${ }^{2, \star}$, Lingzhi Zhao ${ }^{2}$, Suzanne E. Hickman ${ }^{2}$, Terry K. Means ${ }^{2}$, Lindsay Puckett ${ }^{2}$, Dorit Farfara ${ }^{1}$, Nathan D. Kingery ${ }^{2}$, Howard L. Weiner ${ }^{3} \&$ Joseph El Khoury ${ }^{2,4}$

In Alzheimer's disease, soluble amyloid- $\beta$ causes synaptic dysfunction and neuronal loss. Receptors involved in clearance of soluble amyloid- $\beta$ are not known. Here we use short hairpin RNA screening and identify the scavenger receptor Scara1 as a receptor for soluble amyloid- $\beta$ expressed on myeloid cells. To determine the role of Scara1 in clearance of soluble amyloid- $\beta$ in vivo, we cross Scara1 null mice with PS1-APP mice, a mouse model of Alzheimer's disease, and generate PS1-APP-Scara1-deficient mice. Scara1 deficiency markedly accelerates $A \beta$ accumulation, leading to increased mortality. In contrast, pharmacological upregulation of Scara1 expression on mononuclear phagocytes increases $A \beta$ clearance. This approach is a potential treatment strategy for Alzheimer's disease.

\footnotetext{
${ }^{1}$ Department of Neurobiology, Sagol School of Neuroscience, George S. Wise Faculty of Life Sciences, Tel Aviv University, Tel Aviv 699788 , Israel. ${ }^{2}$ Center for Immunology and Inflammatory Diseases, Charlestown, Massachusetts 02129, USA. ${ }^{3}$ Center for Neurologic Diseases, Brigham and Women's Hospital, Harvard Medical School, Boston, Massachusetts 02115, USA. ${ }^{4}$ Division of Infectious Diseases, Massachusetts General Hospital, Harvard Medical School, CNY 149, Room 8301, 149 13th Street, Charlestown, Massachusetts 02129, USA. * These authors contributed equally to this work. Correspondence and requests for materials should be addressed to J.E.K. (email: jelkhoury@partners.org) or to D.F. (email: dfrenkel@tauex.tau.ac.il).
} 
W ork in transgenic mouse models of Alzheimer's disease (AD) indicates that mononuclear phagocytes can promote the clearance of amyloid- $\beta$ (A $\beta)$ peptides. Reduction in the number of recruited mononuclear phagocyte led to accelerated $\mathrm{A} \beta$ deposition especially around blood vessels, leading to intracerebral haemorrhage and increased mortality in a mouse model of $\mathrm{AD}^{1}$. In support of these findings, depletion of perivascular macrophages significantly increased the number of Thioflavin S-positive cortical blood vessels and total perivascular $\mathrm{A} \beta$ levels $^{2}$. Conversely, stimulation of perivascular macrophage turnover or enhancing recruitment of peripheral monocytes reduced cerebral perivascular and parenchymal $A \beta$ load $^{2,3}$. These data suggest that mononuclear phagocytes may delay progression of $\mathrm{AD}$ by promoting clearance of $\mathrm{A} \beta$ and preventing senile plaque formation. However, persistent $A \beta$ accumulation in spite of increasing mononuclear phagocyte numbers in $\mathrm{AD}$ suggests that the ability of these cells to clear $A \beta$ may decrease with age and progression of $\mathrm{AD}$ pathology 4 .

Much attention has been focused for many years on the insoluble aggregates of $\mathrm{A} \beta$ found in senile plaques. Insoluble fibrillar aggregates are neurotoxic in vitro and activate microglia and astrocytes to produce cytokines, chemokines and reactive oxygen and nitrogen species 5 , however, the number of senile plaques in any particular areas of the brain does not always correlate with neuronal death or synaptic loss in that area ${ }^{6-8}$. Such discrepancy brought attention to other non-aggregated forms of $A \beta^{9,10}$. These soluble $A \beta s$ (sA $\beta$ s) include $A \beta$ monomers or oligomers that remain soluble in aqueous buffers after highspeed ultracentrifugation $^{9,10}$. sA $\beta$ may start accumulating in the brain before formation of visible senile plaques. $s A \beta$ is associated with cognitive dysfunction in transgenic mice, and several groups confirmed that a strong correlation exists between sA $\beta$ levels and synaptic loss and neuronal degeneration ${ }^{6-12}$. It is not known whether sA $\beta$ interacts with monocytes, microglia or the potential receptors involved in such interactions. However, because sA $\beta$ starts accumulating before formation of senile plaques and microglia are constantly surveying their environment for any potential injurious stimuli ${ }^{13}$, we hypothesized that mononuclear phagocytes can recognize and bind sA $\beta$.

Microglia and macrophages express several receptors that can promote binding and/or phagocytosis of fibrillar $A \beta$ aggregates in vitro. These include the class $\mathrm{A} 1$ scavenger receptors (Scara1) ${ }^{14}$, the class B2 scavenger receptors (Scarb2) or CD36 (refs 5,15), RAGE ${ }^{16}$ and others. Mononuclear phagocytes also express a number of $A \beta$-degrading enzymes, such as insulysin, neprilysin and others ${ }^{4,17}$. The exact role of each of these receptors in development or progression of $\mathrm{AD}$ is not known. We found that as transgenic $\mathrm{AD}$ mice age, expression of these $\mathrm{A} \beta$ phagocytic receptors and $A \beta$-degrading enzymes decreased significantly in microglia, thereby promoting $A \beta$ accumulation and contributing to neurodegeneration ${ }^{4}$. This led us to hypothesize that restoring the ability of mononuclear phagocytes to phagocytose or degrade $A \beta$ may be a therapeutic modality to delay or stop the progression of $\mathrm{AD}$. In support of this hypothesis, genetic downregulation of the transforming growth factor- $\beta$-Smad $2 / 3$ innate immune signalling pathway, led to increased infiltration of macrophages around blood vessels, and decreased $\mathrm{A} \beta$ levels and behavioural deficits in $\mathrm{AD}$ mice ${ }^{3}$. It is not known if pharmacological stimulation of the mononuclear phagocyte $A \beta$ clearance pathways will also lead to mitigation of AD-like pathology.

In this paper, we use a short hairpin RNA (shRNA) screen to identify receptors for $s A \beta$ and show that Scaral is a phagocytic receptor that mediates clearance of $\mathrm{sA} \beta$ in vitro, and that Scaral deficiency is associated with increased early mortality and $A \beta$ deposition in the PS1-APP mouse model of AD. We also show that pharmacological upregulation of Scara1 leads to increased $\mathrm{A} \beta$ clearance, suggesting that this approach may be a strategy for treatment of $\mathrm{AD}$.

\section{Results}

shRNA screen identifies Scaral as a receptor for sA $\beta$ uptake. To identify mononuclear phagocyte receptors involved in the uptake of sA $\beta$, we used a mouse shRNA library targeting innate immune receptors to screen for genes that mediate macrophage uptake of sA $\beta$ labelled with the fluorescent dye Hilyte-Fluor 488 . We confirmed that our Hilyte-Fluor $488 \mathrm{~A} \beta$ is soluble using native electrophoresis and Thioflavin S staining (Supplementary Fig. S1a,b) as described ${ }^{5-8}$. The RNAi Consortium has previously published that its lentiviral library silences genes in a diverse set of cell types, contains five shRNA for nearly every gene in the murine genome, and is capable of significantly reducing target mRNA levels (Fig. 1a) ${ }^{18}$. Using a subset of the mouse lentiviral shRNA library targeting toll-like receptors, scavenger receptors and other pattern-recognition receptors ${ }^{19}$, we performed a screen to identify genes that mediate macrophage uptake of sA $\beta 1-42$ Hilyte-Fluor 488 using RAW 264.7 (RAW) macrophages.
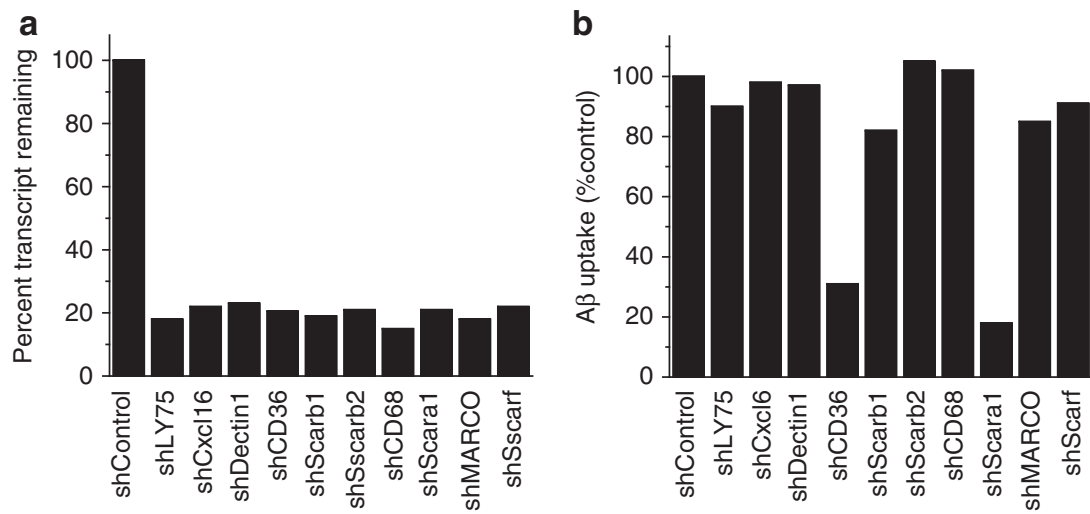

Figure 1 | A shRNA screen identifies Scara1 as a receptor for sA $\boldsymbol{\beta}$. (a) RAW macrophages were transduced with lentivirus encoding gene-specific shRNAs to 30 pattern-recognition receptors or with a control shRNA directed against green fluorescent protein. Gene expression was measured by qPCR, and 3-5 individual shRNAs per gene were identified that significantly reduced mRNA expression. (b) Pattern-recognition receptor expression was knocked down in individual pools of RAW macrophages, which were then incubated with Hilyte-Fluor 488-labelled sA $\beta$ 1- 42 for $2 \mathrm{~h}$. Cell-associated sA $\beta$ was then quantified by flow cytometry. Data are from a representative experiments repeated three times with similar results. 
Silencing expression of all receptors tested was effectively performed to $\sim 20 \%$ of normal expression (Fig. 1a, data not shown). Interestingly, only silencing of the scavenger receptors CD36 and Scaral led to a significant reduction in sA $\beta$ 1-42 Hilyte-Fluor 488 uptake by RAW macrophages (Fig. 1b, data not shown). The results of our shRNA screen indicate that Scaral and CD36 can mediate uptake of sA $\beta$ by mononuclear phagocytes in vitro. Gene silencing of other scavenger receptors, such as Scarb1, Scarf, CD68 and CXCL16 also expressed by macrophages, had no effect on uptake of sA $\beta$ 1-42 Hilyte-Fluor 488 by RAW cells (Fig. 1b), suggesting that Scara1 and CD36 are the main receptors involved in this process.

To confirm that uptake of sA $\beta$ by microglia is indeed a receptor-mediated event, we incubated N9 microglia with increasing concentrations of sA $\beta$ and quantified uptake by flow cytometry. As seen in Fig. 2a, uptake of sA $\beta$ by microglia began to reach saturation at $\sim 2 \mu \mathrm{M}$. In addition, uptake of sA $\beta$ by N9 microglia was time dependent and reached saturation at $\sim 4 \mathrm{~h}$ (Fig. 2b). These data indicate that uptake of sA $\beta$ by microglia is likely to be receptor-mediated and is saturable in a dose- and time-dependent manner. To further confirm that uptake of sA $\beta$ by microglia is mediated by scavenger receptors, we incubated N9 cells for $2 \mathrm{~h}$ with $1 \mu \mathrm{M}$ sA $\beta 1-42$ Hilyte-Fluor $488 \pm 200 \mu \mathrm{M}$ Fucoidan, a broad inhibitor of scavenger receptors, and measured uptake by flow cytometry. Fucoidan significantly inhibited the amount of sA $\beta$ taken by N9 microglia (Fig. 2c) and the number of cells that took sA $\beta$ (Fig. $2 \mathrm{~d}$ ).

Scara1 deficiency is associated with reduced sA $\beta$ uptake. Expression of Scara1 in HEK293 cells is sufficient to promote the ability of these cells to take $A \beta$ (Supplementary Fig. S2). To determine if Scaral is necessary for uptake of sA $\beta$ by primary monocytes and microglia as suggested by our shRNA screen in macrophages, we isolated circulating monocytes and microglia from adult mice with targeted deletion of various scavenger receptors and from wild-type (WT) mice and tested the ability of these freshly isolated monocytes and microglia to take up sA $\beta$. Interestingly, only targeted deletion of Scaral led to a significant reduction in the uptake of sA $\beta$ by fresh monocytes (Fig. 3a) and microglia (Fig. $3 \mathrm{~b}$ ) by $65 \%$ and $50 \%$, respectively. Targeted deletion of CD36 did not affect uptake of sA $\beta$ by monocytes and only reduced its binding to microglia by $25 \%$, which was not statistically significant. These data indicate that Scaral is a major receptor for uptake of $s A \beta$ by monocytes and microglia and that Scara1-mediated uptake is not redundant. The lack of effect of targeted deletion of CD36 on sA $\beta$ uptake suggests that unlike Scara1, CD36 does not have a non-redundant role in clearance of sA $\beta$. Because targeted deletion of CD36 leads to reduced A $\beta$ induced activation of mononuclear phagocytes to produce cytokines and reactive oxygen species, our data suggest that CD36 may be involved in the activation of mononuclear phagocytes by sA $\beta$ and not in the clearance of $s A \beta$ by these cells $s^{5,20,21}$. This is reminiscent of the role that $\mathrm{CD} 36$ has in the interaction between macrophages and oxidized low-density lipoproteins (LDLs) ${ }^{22}$.

Increased mortality in PS1-APP-Scara1 ${ }^{-/-}$mice. To determine if Scaral mediates clearance of sA $\beta$ in vivo, and the effects of such clearance on accumulation of A $\beta$, we generated PS1-APPScara ${ }^{-1-}$ mice and analysed these mice for $A \beta$ levels and $A D$ like pathology. To accomplish this, we utilized a strategy that we have successfully used in the past to generate transgenic APP mice deficient in the chemokine receptor Ccr2 (ref 1). Briefly, PS1-APP
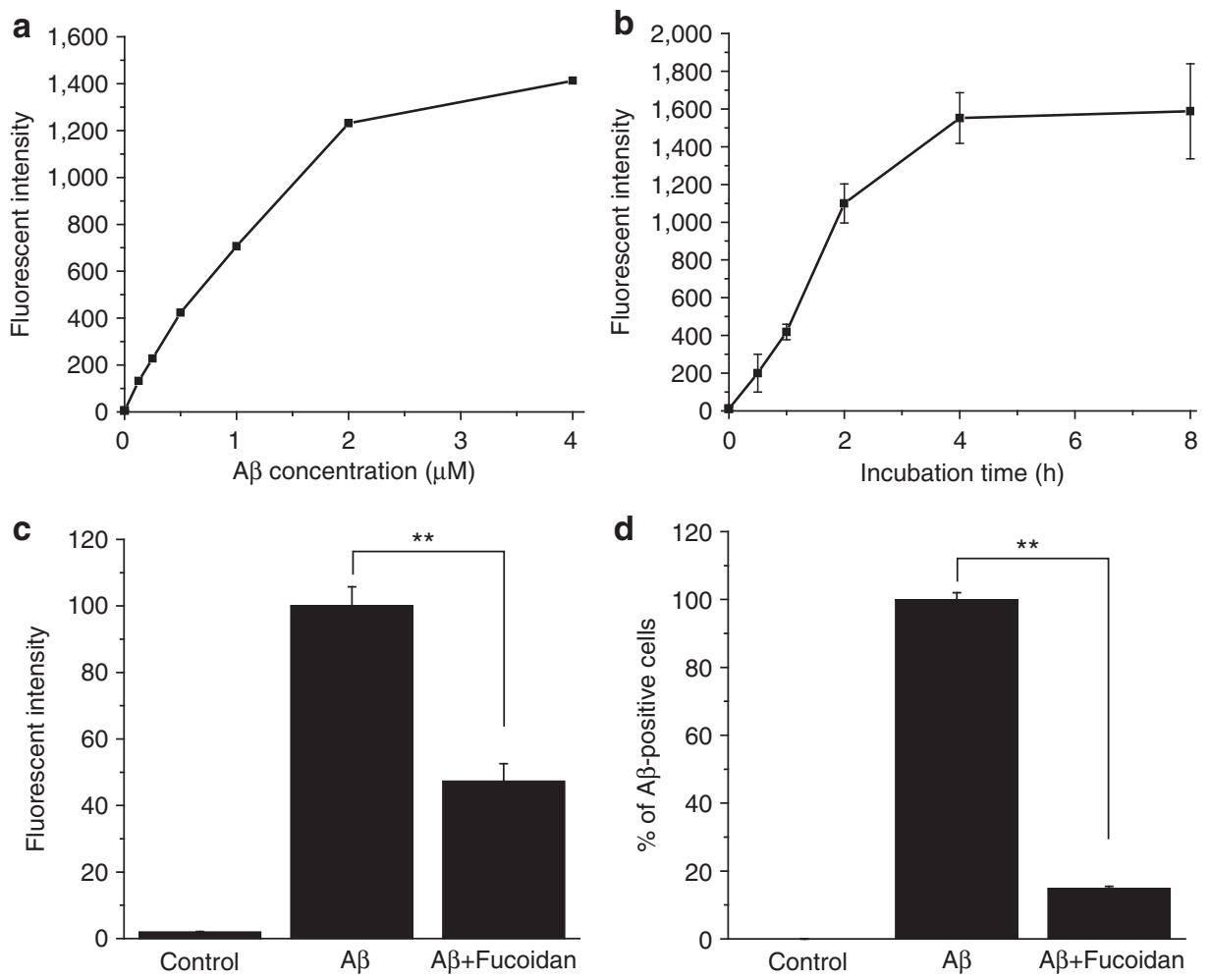

Figure 2 | Uptake of sA $\boldsymbol{\beta}$ by $\mathbf{N 9}$ microglia. (a,b) Uptake of Hilyte-Fluor 488-labelled sA $\beta$ by N9 microglia is saturable in a dose- and time-dependent manner. (c,d) N9 cells were pretreated with $200 \mu \mathrm{g} \mathrm{ml}{ }^{-1}$ Fucoidan, a broad inhibitor of scavenger receptors, and then incubated with $1 \mu \mathrm{M}$ freshly dissolved Hilyte-Fluor 488-labelled sA $\beta$ 1-42 for $2 \mathrm{~h}$. The uptake of sA $\beta$ was measured by flow cytometry. Fucoidan significantly inhibited the amount of sA $\beta$ taken up by $N 9$ cells and the number of cells that took $s A \beta$, indicating that microglial scavenger receptors are essential for phagocytosis of soluble $A \beta$. Values are mean \pm s.e.m. $n=3$ (analysis of variance, ${ }^{\star \star} P<0.05$ ). 

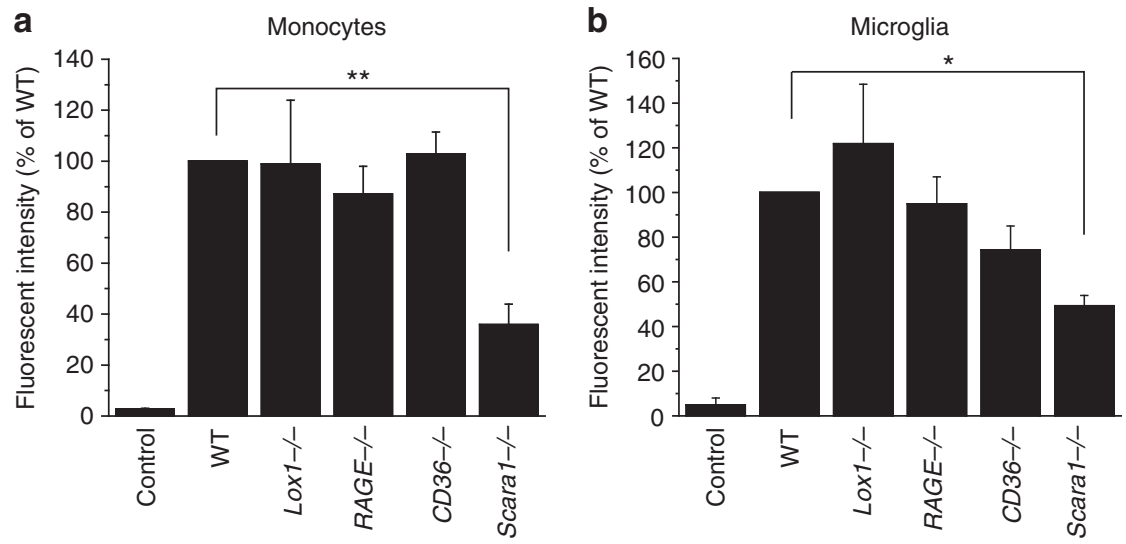

Figure 3 | Scara1 is a major monocyte and microglial receptor for sA 3 . Targeted deletion of Scara1, but not CD36, LOX-1 or RAGE, significantly reduces $\mathrm{sA} \beta$ uptake by CD11b + monocytes (a) and microglia (b). Monocytes and microglia isolated from C57BL6 mice or mice with targeted deletion of the indicated gene were incubated with $1 \mu \mathrm{M}$ Hilyte-Fluor 488 -labelled sA $\beta 1-42$ for $2 \mathrm{~h}$, and uptake of sA $\beta$ was measured by flow cytometry. Data points represent the mean of triplicate determinations \pm s.e.m., $n=4$ (analysis of variance, ${ }^{\star} P<0.05,{ }^{\star \star} P<0.03$ ).

a
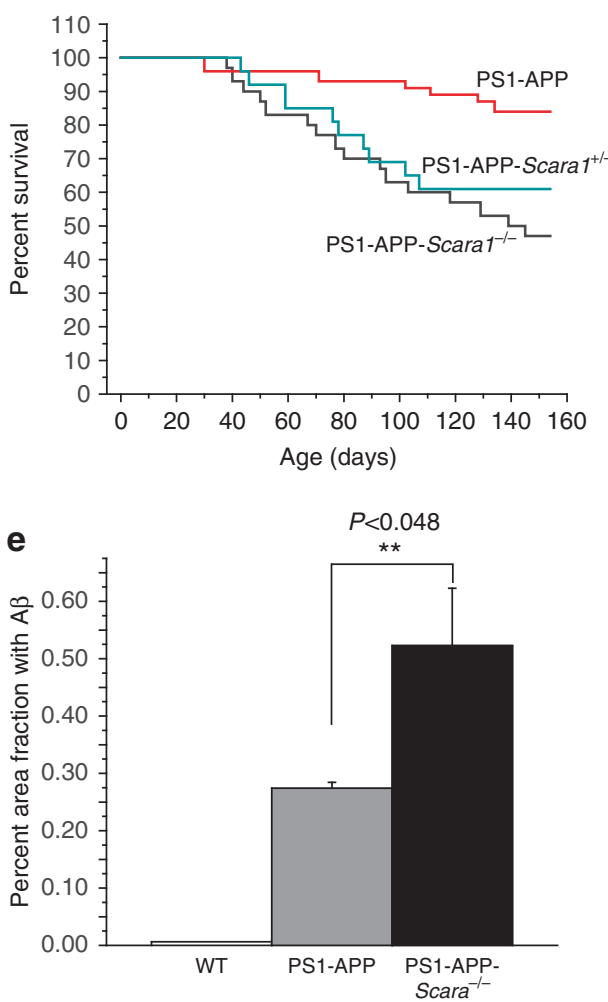

b

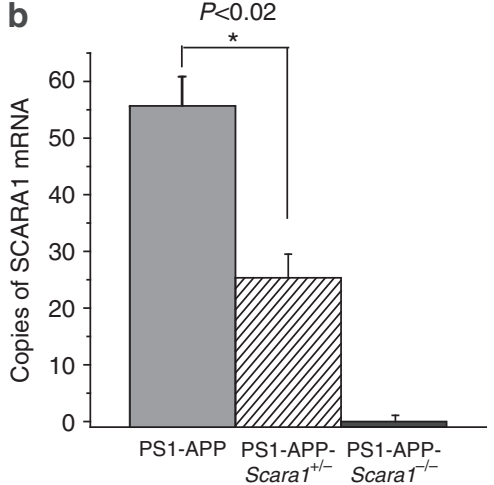

f

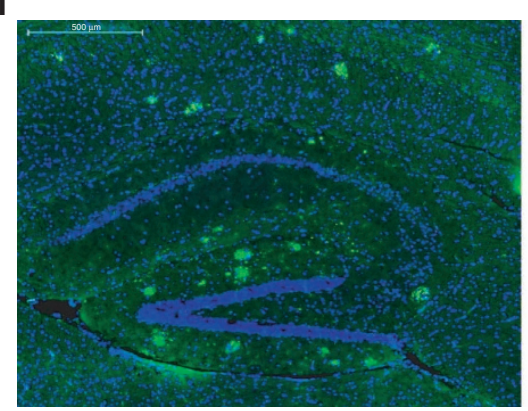

PS1-APP

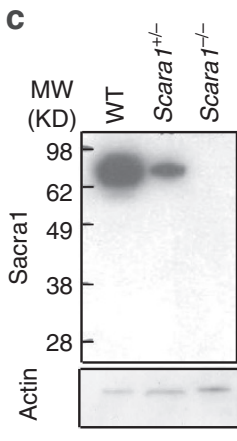

d

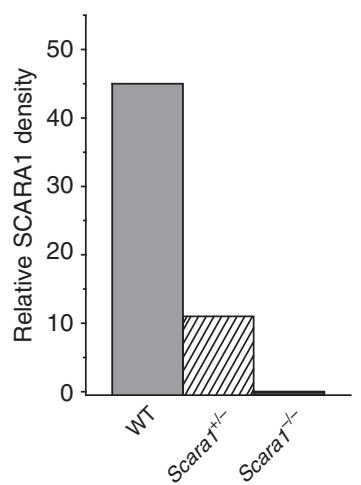

g

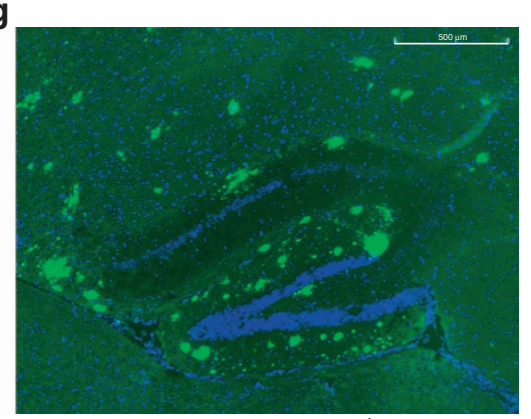

PS1-APP-Scara ${ }^{-1-}$

Figure 4 | Scara1 deficiency is associated with increased mortality and higher A $\boldsymbol{\beta}$ deposition in PS1-APP mice. (a) Survival curves for PS1-APP ( $n=30$ ), PS1-APP-Scara1 ${ }^{-1+}(n=25)$ and PS1-APP-Scara1 ${ }^{-1-}(n=24)$ mice. (b) Level of Scara1 mRNA in PS1-APP-Scara1 ${ }^{+/-}$mice is $45 \%$ that of PS1-APP mice. Reduced gene dosage in Scara1 ${ }^{+/-}$mice is associated with a 76\% reduction in Scara1 protein levels as shown in this representative western blot (c) and quantified by densitometry (d); representative blot and quantification were repeated three different times with similar results. (e-g) Scara1 deficiency significantly increases A $\beta$ deposition in 8-month-old PS1-APP mice. Increased number of visible A $\beta$ deposits in PS1-APP-Scara ${ }^{-/-}$brains (g) compared with PS1-APP brains (f). Scale bars, $500 \mu \mathrm{m}$. Data points represent the average of five independent determinations per mouse, \pm s.e.m., $n=4-6$ mice per group (analysis of variance, ${ }^{\star} P<0.02,{ }^{* \star} P<0.048$ ).

mice on a C57BL6 background were crossed to Scara ${ }^{-1-}$ mice (also on a C57BL6 background) to generate PS1-APP-Scara $1^{+1-}$ mice. We then crossed these mice with $S_{c a r a 1}{ }^{-/-}$mice to generate PS1-APP-Scara1 ${ }^{-1}$ mice. PS1-APP-Scara1 ${ }^{+1-}$ and PS1-APP-Scara1 ${ }^{-/-}$mice initially appeared healthy, without obvious behavioural abnormalities. However, after reaching 7 weeks of age, there was a marked increase in the mortality rate of both PS1-APP-Scaral ${ }^{+1-}$ and PS1-APP-Scara1-Imice as compared with control PS1-APP mice (Fig. 4a). By the age of 160 days, 39\% of PS1-APP-Scara $1^{-/+}$and $53 \%$ of 
PS1-APP-Scaral ${ }^{-1-}$ mice had died, compared with $16 \%$ of PS1APP mice. The number of dying PS1-APP-Scara ${ }^{+/-}$mice reached a plateau at age 108 days, whereas PS1-APP-Scara ${ }^{-1-}$ mice continued to die until our data collection had stopped by age 160 days. And while PS1-APP-Scara1 ${ }^{-/-}$mice had higher mortality at 160 days than PS1-APP-Scara $1^{+/}$mice, the overall difference between those two genotypes did not reach statistical significance (Fig. 4a). These data show that Scaral deficiency leads to increased mortality in mice expressing the PS1-APP transgenes. These data are similar to what we found with Ccr2 deficiency (a defect that leads to reduced mononuclear phagocyte recruitment but does not affect their function ${ }^{1}$ ). These data indicate that blocking mononuclear phagocyte number or function has detrimental effect in AD mouse models.

As expected, Scara1 mRNA levels in the brains of PS1-APPScara $1^{+l-}$ mice were $\sim 45 \%$ of those in PS1-APP mice and were not detectable in PS1-APP-Scara $1^{-/-}$mice as determined by quantitative real-time PCR (qPCR) (Fig. 4b). A similar trend was observed in WT, Scara1 ${ }^{+/}-$and Scara1 ${ }^{-I-}$ mice (not shown). Interestingly, analysis of Scara1 protein expression by western blot showed that 55\% reduction in Scara1 gene dosage (as occurs in Scaral ${ }^{+/-}$mice) is associated with a much larger $76 \%$ reduction in Scara1 protein level (Fig. 4 c,d). Because survival of PS1-APP mice appears to correlate with Scaral expression, it is possible that efficient clearance of $A \beta$ requires a certain threshold of Scaral expression, and the disproportionate reduction in Scara1 protein levels in Scara1 ${ }^{+/}$mice, likely below such threshold, may explain the comparable mortality rates between PS1-APP-Scara1 ${ }^{+/-}$and PS1-APP-Scara1 ${ }^{-1-}$ mice.

Increased A $\beta$ accumulation in PS1-APP-Scara1 ${ }^{-/-}$mice. To determine the effects of Scaral deficiency on AD-like pathology, we analysed the brains of 8-month-old PS1-APP-Scara $1^{-9}$ mice for A $\beta$ deposition in comparison with PS1-APP mice. As seen in Fig. 4e-g, Scaral deficiency led to a significant increase in the surface area fraction of the brain stained for A $\beta$ in PS1-APP mice. As expected, WT mice did not have any A $\beta$ in their brain at this age (Fig. 2e). These data support the results obtained from our shRNA screen and from the experiments showing decreased uptake of sA $\beta$ in vitro by monocytes and microglia isolated from Scara1 ${ }^{-1-}$ mice (Fig. 3a,b). The data further indicate that Scara1 is a major receptor involved in endogenous clearance of $A \beta$ and that deficiency in Scara1, as occurs in aging $\mathrm{AD}$ mice ${ }^{4}$, is associated with increased $A \beta$ levels.

Our shRNA-screening data showed that CD36 can mediate uptake of sA $\beta$. RAGE has been also shown to be a receptor for $\mathrm{A} \beta^{16}$. To determine if Scara1 deficiency indirectly affected CD36 or RAGE expression, we measured CD36 and RAGE mRNA in young (150 days) WT, Scara1 ${ }^{-/-}$, PS1-APP and PS1-APPScara $1^{-1-}$ brains by qPCR. As seen in Fig. 5a, Scaral deficiency did not affect CD36 mRNA levels in Scara1 ${ }^{-1-}$ or PS1-APPScara $1^{-1-}$ brains. Furthermore, we did not detect any difference in expression of RAGE in PS1-APP-Scara $1^{-1}$ - mice compared with PS1-APP mice (Not shown). These data indicate that increased A $\beta$ deposition in PS1-APP-Scara1 ${ }^{-1-}$ brains is likely due to decreased Scara1-mediated A $\beta$ clearance in these mice and not due to a change in expression of other $A \beta$ receptors such as Cd36 and RAGE.

$A \beta$ levels are also regulated by several $A \beta$-degrading enzymes such as neprilysin and insulysin ${ }^{17}$. To determine if increased $A \beta$ levels in the brains of PS1-APP-Scara $1^{-1-}$ mice are associated with reduced expression of neprilysin and/or insulysin, we measured mRNA levels of these two enzymes in the brains of young (150 days) WT, Scara1 ${ }^{-/-}$, PS1-APP and PS1-APPScara $1^{-1-}$ mice. We found that expression of insulysin and neprilysin was comparable in WT and Scara1 ${ }^{-1}$ mice. Interestingly, expression of neprilysin was significantly higher in young PS1-APP mice compared with WT mice, suggesting that early in the disease process, uptake of $A \beta$ may be associated with increased $A \beta$ degradation. Expression of insulysin was also slightly higher in PS1-APP mice compared with WT mice, but the difference was small and did not reach statistical significance (Fig. 5b,c). In contrast, both neprilysin and insulin expression was significantly reduced by $38 \%$ and $36 \%$, respectively, in PS1-APPScara $1^{-1}$ brains as compared with PS1-APP brains (Fig. 5b,c). These data suggest that phagocytosis of A $\beta$ in young PS1-APP mice is associated with increased $A \beta$ degradation, whereas decreased uptake of $A \beta$, as occurs in PS1-APP-Scara ${ }^{-/-}$ mice, leads to decreased $A \beta$ degradation and further increase in $\mathrm{A} \beta$ accumulation, thereby initiating a self-propagating loop of events that lead to accelerated disease progression.

Data shown in Fig. 4a indicate that mortality of PS1-APPScara $1^{+1-}$ mice was only slightly better than that of PS1-APPScaral ${ }^{-1-}$, but the difference did not reach statistical significance. To determine if partial Scaral deficiency also affected
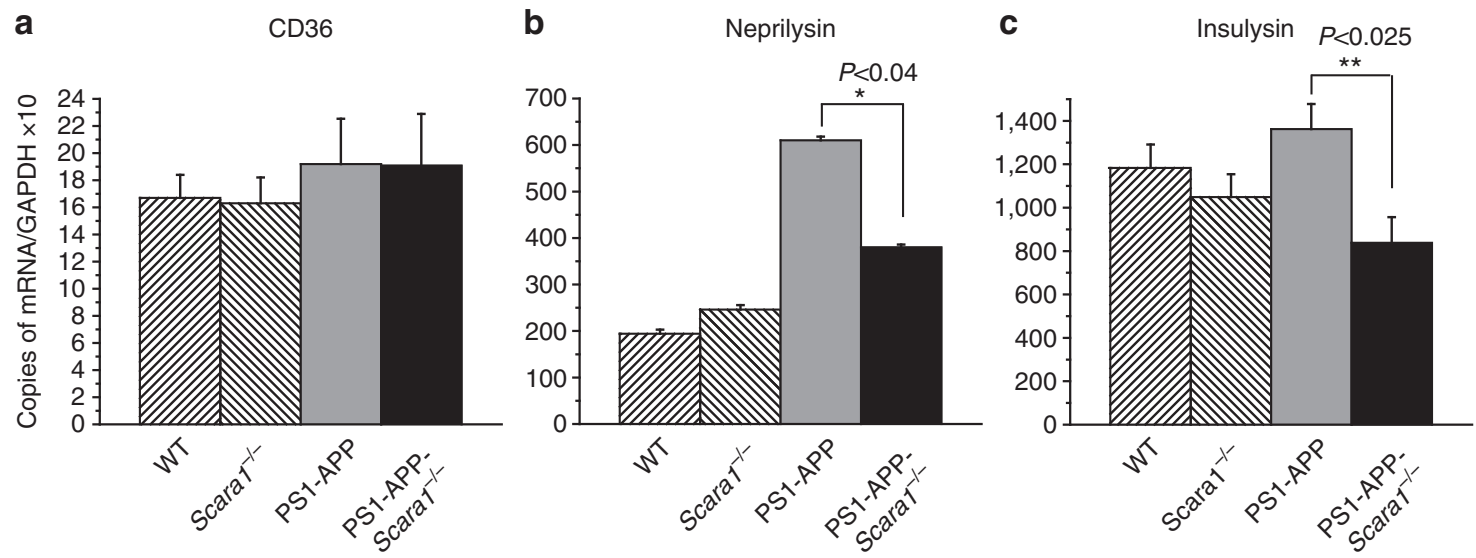

Figure 5 | Effect of Scara1 deficiency on CD36 and Aק-degrading enzymes. WT and Scara1-/- mice express comparable mRNA levels of CD36 (a), and the A $\beta$-degrading enzymes neprilysin (b) and insulysin (c) in their brains. In contrast, PS1-APP-Scara ${ }^{-/-}$mice express similar mRNA levels of CD36 as compared with PS1-APP mice (a), but significantly lower levels of neprilysin (b) and insulysin (c). Each bar is the average of three triplicate determinations per mouse from 3-5 different mice per genotype \pm s.e.m. (analysis of variance, ${ }^{\star} P<0.04,{ }^{\star \star} P<0.025$ ). GAPDH, glyceraldehyde 3-phosphate dehydrogenase. 
AD-like pathology, we analysed the brains of young (150 days) PS1-APP-Scara1 ${ }^{+1-}$ mice for A $\beta$ deposition in comparison with PS1-APP mice. As seen in Supplementary Fig. S3a, PS1-APPScara $1^{+/-}$mice exhibited a significant increase in $\mathrm{A} \beta$ levels as measured by enzyme-linked immunosorbent assay (ELISA) $\left(700 \mathrm{pg} \mathrm{mg}^{-1}\right.$ brain protein versus $1500 \mathrm{pg} \mathrm{mg}^{-1}$ protein, $P<0.002)$. This increase also correlated with increased visible A $\beta$ deposits (Supplementary Fig. S3b,c).

Of note, the number of visible $A \beta$ deposits in $\sim 150$-day-old PS1-APP and PS1-APP-Scara1 ${ }^{+/-}$mice were small (Supplementary Fig. S3b,c), and we did not detect any Thioflavin S-positive deposits (not shown), suggesting that the majority of A $\beta$ measured in these mice is in soluble or non-aggregated form. These data support the results obtained from our shRNA screen and from the experiments showing decreased uptake of sA $\beta$ in vitro by monocytes and microglia isolated from Scara1deficient mice.

Upregulation of Scaral leads to increased A $\beta$ clearance. Protollin, a proteosome-based adjuvant comprising purified outer-membrane proteins of Neisseria meningitides and lipopolysaccharide that is well tolerated in humans ${ }^{23}$, promotes increased accumulation of mononuclear phagocytes and increased clearance of amyloid when given intranasally to transgenic $\mathrm{AD}$ mice ${ }^{24,25}$. We investigated whether the mechanism by which Protollin enhances $A \beta$ uptake by mononuclear phagocytes involves upregulation of Scaral or CD36. As seen in Fig. 6a-c, although Protollin upregulated mRNA levels of Scaral by more than threefold, it did not affect mRNA levels of CD36. Furthermore, Protollin upregulated $\mathrm{Ccl} 2$ mRNA levels by $\sim 7$ fold (Fig. $6 \mathrm{~d}$ ). These data suggest that the increased numbers of mononuclear phagocytes observed in Protollin-treated Alzheimer's mice are may be because of their recruitment

to sites of $\mathrm{A} \beta$ deposition via $\mathrm{Ccl} 2$. These data also suggest that Protollin-induced enhanced $A \beta$ clearance may in part be due to increased recruitment of mononuclear phagocytes to A $\beta$-deposition sites.

Clearance of $\mathrm{A} \beta$ deposits in situ from brain sections derived from transgenic $\mathrm{AD}$ mice has been used as a surrogate measure for astrocyte ability to clear $\mathrm{A} \beta$ in vivo ${ }^{26}$. To test if Protollininduced activation of $\mathrm{N} 9$ microglia promotes the ability of these cells to reduce $A \beta$ deposits, we incubated these cells with brain sections derived from aged transgenic PS1-APP brains in the

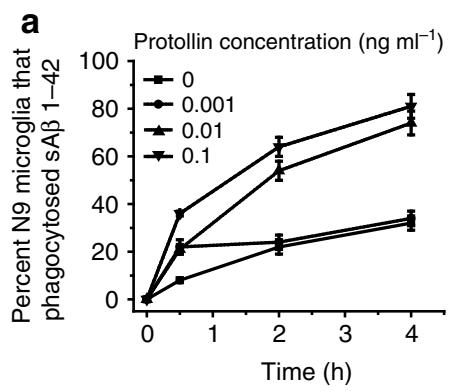

\section{b}

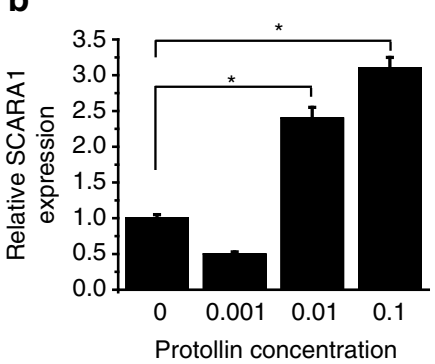

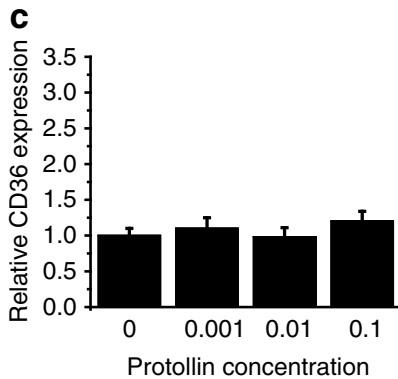
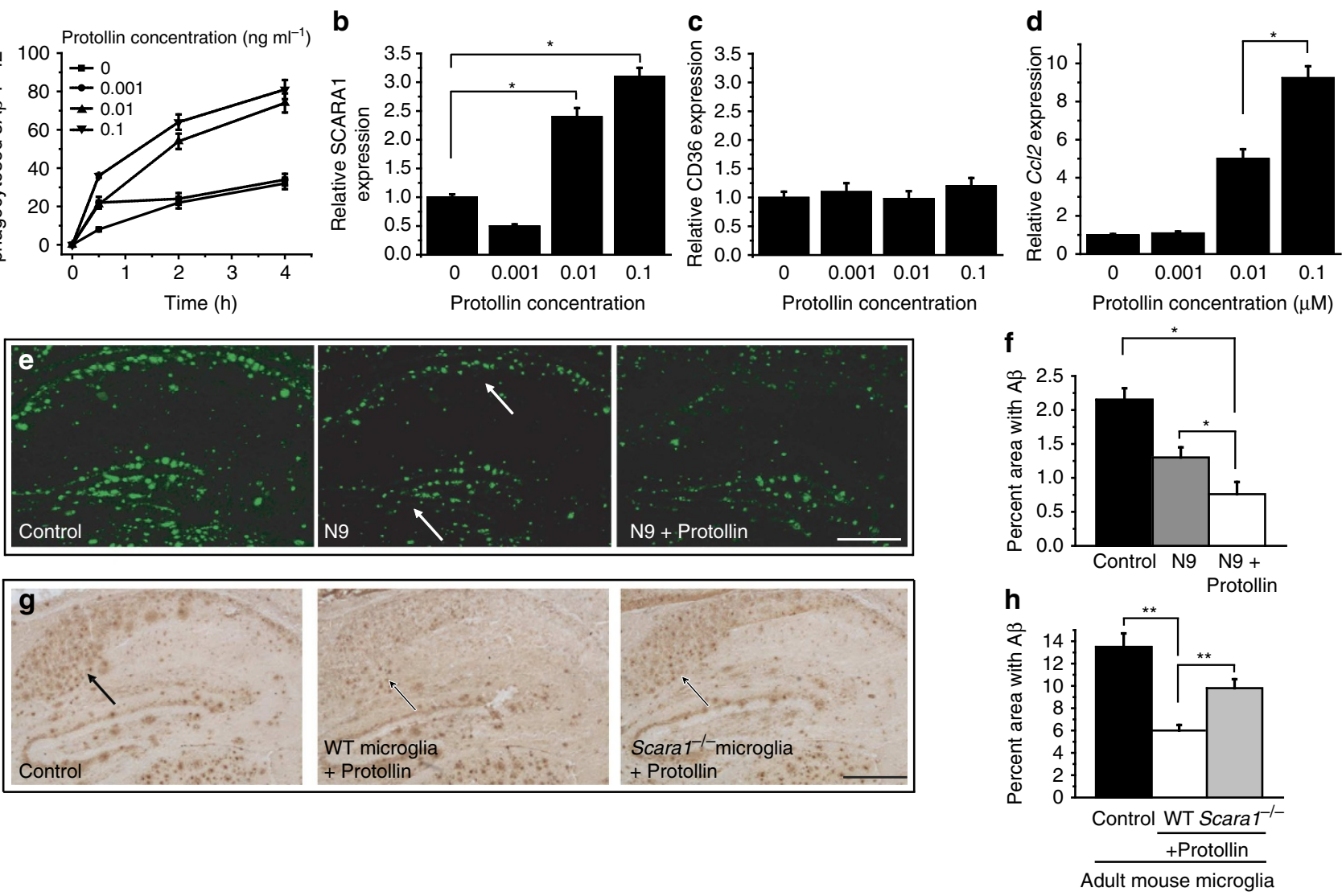

Figure 6 | Upregulation of Scara1 increases sA $\beta$ uptake in vitro and in situ in a Scara1-dependent mechanism. (a) Protollin, a proteosome-based adjuvant comprising purified outer-membrane proteins of $N$. meningitides and lipopolysaccharide that is well tolerated in humans, upregulates sA $\beta$ uptake in N9 microglia. Cells were incubated for $18 \mathrm{~h}$ in presence of different concentrations of Protollin, and incubated with $0.5 \mu \mathrm{g} \mathrm{ml} \mathrm{I}^{-1} \mathrm{sA} \beta 1-42$, Hilyte-Fluor 488-labelled, for $30 \mathrm{~min}, 2 \mathrm{~h}$ and $4 \mathrm{~h}$ incubation. Cell-associated sA $\beta$ was measured by flow cytometry. (b,c) Protollin upregulates Scara1 but not CD36 expression at the transcriptional level as measured by qPCR. (d) Protollin upregulates expression of C 12 in a dose-dependent manner. N9 microglia were incubated with the indicated concentration of Protollin for $24 \mathrm{~h}$, and expression of Scara1, CD36 or Ccl2 was quantified by qPCR. (e,f) N9 microglia cells are plated on brain sections from a mouse model of $A D$ for $24 \mathrm{~h} \pm$ Protollin. The percent of the surface area of the brain covered by $A \beta$ was measured by staining with anti-A $\beta$ antibodies. ( $\mathbf{g}, \mathbf{h})$ Reduction of amyloid load in the hippocampus following $24 \mathrm{~h}$ incubation with microglia is Scara1 dependent. Adult microglia isolated from WT and Scara1 ${ }^{-/-}$mice were added to brain sections from a mouse model of AD and incubated for $24 \mathrm{~h} \pm$ Protollin. The percent of the surface area of the brain covered by $A \beta$ was measured by staining with anti-A $\beta$ antibodies. Scale bars, $500 \mu \mathrm{m}$. All data points represent the average of four independent determinations \pm s.e.m. (analysis of variance, ${ }^{\star} P<0.05$, ${ }^{\star} * P<0.03$ ). 
presence of increasing concentrations of Protollin. Such sections normally exhibit florid $A \beta$ deposits in the cortex and hippocampus. As seen in Fig. 6e,f, the addition of Protollinstimulated N9 microglia significantly reduced the size and number of $A \beta$ deposits in such sections in the hippocampus when compared with untreated sections or sections incubated with unstimulated N9 microglia. To determine the relative importance of Scaral in A $\beta$ clearance by microglia activated with Protollin, we isolated WT and Scara $1^{-1-}$ adult microglia as previously described ${ }^{26}$ and compared their ability to clear brain A $\beta$ deposits from PS1-APP brain slices as we did with N9 cells. After incubation of the brain sections with microglia from WT mice for $72 \mathrm{~h}$, there was a $58 \%$ reduction in total $A \beta$ deposits in the hippocampus $(P=0.0013)$ as compared with untreated sections and sections incubated with microglia from Scara ${ }^{-1-}$ mice $(P=0.0214)$, which showed only a $20 \%$ reduction $(P=0.0317)$ as compared with untreated section (Fig. 6g,h). These data strongly support that Protollin-induced increased A $\beta$ clearance is in part dependent on Scaral and that pharmacological upregulation of Scaral expression can be successfully accomplished.

\section{Discussion}

The role of mononuclear phagocytes including peripheral monocytes and microglia in $\mathrm{AD}$ is subject to intense investigation $^{27}$. Although a shotgun approach that deletes all microglia for a brief period of time did not show an effect on amyloid deposition in a transgenic $\mathrm{AD}$ mouse model ${ }^{28}$, targeted genetic manipulation of the innate immune system in mouse models of $\mathrm{AD}$ provided strong evidence that mononuclear phagocytes have critical roles in regulating $A \beta$ deposition by regulating the $A \beta$ clearance pathways. Indeed, defects in microglial/mononuclear phagocyte accumulation in transgenic APP mice and other mouse models of $\mathrm{AD}$ are associated with increased $\mathrm{A} \beta$ deposition and increased mortality and/or cellular toxicity in these mice $e^{1,29}$. Furthermore, deficiencies and/or mutations in specific innate immune receptors such as CD14 and TLR4 affect A $\beta$ deposition ${ }^{30,31}$. Unfortunately, with aging and progression of $\mathrm{AD}$, innate immune cells of the brain appear to become dysfunctional, and their ability to clear amyloid becomes significantly reduced ${ }^{4}$. The exact mechanisms of such microglial dysfunction are not clear, but are believed to be at least, in part, due to downregulation of microglial $A \beta$ phagocytic receptors ${ }^{4}$. In this manuscript, we provide direct evidence that the microglial/ monocyte scavenger receptor Scaral is a key $A \beta$ clearance receptor expressed by these cells and that genetic deficiency in Scaral is indeed associated with increased accumulation of $A \beta$. In addition, deficiency in Scaral is associated with increased mortality in PS1-APP mice. Such increases in mortality and A $\beta$ deposition are similar to what we observed in APP-Ccr2-deficient mice, and suggest that abnormalities in the number and/or function of mononuclear phagocytes can have detrimental effects on transgenic mouse models of AD. In contrast to the detrimental effects associated with dysfunction of the innate immune system in $\mathrm{AD}$, we also show that restoring some of the $\mathrm{A} \beta$-clearing ability of the innate immune system by pharmacological upregulation of Scaral leads to increased $A \beta$ clearance by mononuclear phagocytes and microglia, and therefore may be beneficial in slowing down or delaying progression of this disease. It is not clear if upregulating Scaral expression by non-pharmacological approaches will also lead to increased sA $\beta$ clearance. Indeed, upregulating Scaral mRNA in aging $\mathrm{AD}$ mice following irradiation does not seem to have the same effect as using Protollin $^{32}$. It is possible that such apparent discrepancy may be due to increased influx of $A \beta$ across the blood brain barrier as a result of increased permeability of the blood brain barrier following irradiation, to differences in the age and genotype of the mice analysed and/or the approach used to quantify sA $\beta$. Nonetheless, our data clearly indicate that upregulating Scara1 expression pharmacologically is a viable therapeutic strategy to treat $\mathrm{AD}$.

We showed previously that blocking CD36-A $\beta$ interactions reduces $A \beta$-induced activation of microglia to produce cytokines and reactive oxygen species 5,20 . We also showed that blocking these interactions does not affect phagocytosis of $A \beta$. Therefore, data from these papers indirectly support our findings in this manuscript that Scara1, rather than CD36, is the main receptor for clearance of $\mathrm{A} \beta$ by mononuclear phagocytes. Our data also suggest that Scara1 and CD36 have complementary nonredundant roles in the interactions of mononuclear phagocytes with $A \beta$. Scara1-A $\beta$ interactions are beneficial and promote phagocytosis and clearance of $\mathrm{A} \beta$, whereas $\mathrm{CD} 36-\mathrm{A} \beta$ interactions are harmful and lead to production of neurotoxins and proinflammatory molecules. This is reminiscent of the role that CD36 has in the interaction between macrophages and oxidized LDLs in atherosclerosis. Indeed, we have shown in the past that while Scara1 mediates adhesion to oxidized LDL-coated surfaces, CD36 mediates macrophage activation by oxidized LDL to produce reactive oxygen species ${ }^{22}$. In this regard, the findings presented in this manuscript draw an exciting, not yet described, parallel between atherosclerosis and AD. Dissecting the complex roles of various scavenger receptors in mononuclear phagocyte$\mathrm{A} \beta$ interactions is therefore very important and, as we are showing in this manuscript, has therapeutic implications for AD.

\section{Methods}

shRNA lentiviral infections. Plasmids encoding lentiviruses expressing shRNAs were obtained from the RNAi Consortium library TRC-Mm1.0 (ref. 18), purified using the QiaPrep miniprep kit (Qiagen, Valencia, CA, USA) and then transfected into HEK 293T cells with a three-plasmid system to produce lentivirus. Infection conditions were optimized in 96-well plates. A total of 20,000 macrophages were placed in each well of 96-well tissue culture dishes and infected using $5 \mu \mathrm{l}$ of shRNA lentiviral supernatant and $7.5 \mu \mathrm{g} \mathrm{ml}^{-1}$ polybrene. The cells were spun for $30 \mathrm{~min}$ at 2,000 r.p.m. and incubated for $24 \mathrm{~h}$. To select for shRNA integration, infected cells were placed in puromycin $\left(3 \mu \mathrm{g} \mathrm{ml}^{-1}\right)$ and RPMI containing $10 \%$ fetal bovine serum (FBS). The cells were tested 1-2 weeks after infection.

Mice. PS1-APP bi-transgenic mice (B6C3-Tg (APPswe, PSEN1dE9)85Dbo/J stock number 004462) 33,34 were purchased from The Jackson Laboratories (Bar Harbor ME, USA) and bred in the animal care facilities at Massachusetts General Hospital. Control mice are non-transgenic littermates of those PS1-APP mice expressing these transgenes.

Scaral ${ }^{-1-}$ mice were generated by Professor Tatsuhiko Kodama, and a colony has been maintained in our lab for many years ${ }^{35}$. CD $36^{-1-}$ mice were generated by Dr Kathryn Moore on a C57BL6 background, and a colony has been maintained in our lab for many years ${ }^{5}$. PS1-APP, Scara ${ }^{-1-}$ and CD36 $6^{-1-}$ mice were backcrossed for more than 13 generations on a C57BL/6 background. Lox-1 ${ }^{-1-}$ mice $^{36}$ were a generous gift from Professor Tatsuya Sawamura, National Cardiovascular Center Research Institute Osaka, JAPAN. RAGE ${ }^{-/-}$mice $^{37}$ were a generous gift from Professor Hiroshi Yamamoto, Kanazawa, Japan.

All protocols were approved by the Massachusetts General Hospital and Brigham and Women's Hospital Institutional Animal Care and Use Committees and met the US National Institutes of Health guidelines for the humane care of animals.

Stimulation and cell surface staining of N9 microglia. N9 mouse microglia (a gift from Dr P. Riciarrdi-Castagnoli, University of Milano, Bicocca, Italy) ${ }^{14}$ were plated on 24-well plates coated with $1 \mu \mathrm{g}$ fibronectin per $\mathrm{cm}^{2}$ (Sigma-Aldrich) and grown overnight in RPMI supplemented with $10 \% \mathrm{FBS}$, L-glutamine $(2 \mathrm{mM})$, penicillin $10 \mathrm{IU} \mathrm{ml}^{-1}$ and streptomycin $10 \mu \mathrm{g} \mathrm{ml}^{-1}$. The following day, Protollin (GlaxoSmithKline, Laval, Quebec, Canada) was added and cells were incubated overnight. For staining, cells were lifted from the plate with CellStripper (Mediatech), resuspended in PBS/1\% BSA/2\% FBS containing $10 \mu \mathrm{g} \mathrm{ml}{ }^{-1} \mathrm{Fc}$ block (AbD Serotec) and then APC-labelled anti-mouse CD11b $\left(1 \mu \mathrm{g} \mathrm{ml}^{-1}\right)(\mathrm{BD}$ Biosciences Pharmingen, San Diego, CA, USA), APC-labelled hamster anti-mouse CD36 clone HM36 $\left(1 \mu \mathrm{g} \mathrm{ml}^{-1}\right.$ ) (BioLegend, San Diego, CA, USA), Alexa647labelled anti-CD204 (Scara1) clone $2 \mathrm{~F} 8\left(2.5 \mu \mathrm{g} \mathrm{ml}^{-1}\right)$ (AbD Serotec) or isotype- 
matched control antibodies (same concentrations as primary antibodies) were added and incubated on ice for $1 \mathrm{~h}$. Cells were then fixed, and fluorescence intensity was measured using a FACScalibur (BD Biosciences) flow cytometer.

Isolation of CD11b ${ }^{+}$microglia from adult brains. Adult mouse microglia were isolated as described ${ }^{4}$. Briefly, 12-week-old mice were euthanized and perfused with $30 \mathrm{cc}$ PBS without $\mathrm{Ca}^{+}+$and $\mathrm{Mg}^{+}+\left(\mathrm{PBS}^{-}\right)$. Brains were then rinsed, minced and treated with Dispase and Collagenase Type 3 (Worthington Biochemicals, Lakewood, NJ, USA) for $45 \mathrm{~min}$ at $37^{\circ} \mathrm{C}$, followed by addition of $40 \mathrm{U} \mathrm{ml}^{-1}$ DNase I grade II (Roche Applied Science, Indianapolis, IN, USA) and incubation for an additional $15 \mathrm{~min}$. The enzymes were inactivated by addition of $20 \mathrm{ml}$ Hanks Balanced Salt Solution without $\mathrm{Ca}^{++}$and $\mathrm{Mg}^{++}$(HBSS $=$) (Mediatech) containing $2 \mathrm{mM}$ EDTA and 2\% FBS, and the digested brain bits were triturated sequentially with a 25-, 10- and 5-ml pipette (8-10 times each step) and passed over a $100-\mu \mathrm{m}$ filter (Fisher Scientific, Pittsburgh, PA, USA). Cells were centrifuged and resuspended in RPMI/L-glutamine and mixed with physiological Percoll (Sigma-Aldrich), and centrifuged at $850 \mathrm{~g}$ for $45 \mathrm{~min}$. The cell pellet was resuspended in RPMI, and the cells were passed over a 70- $\mu \mathrm{m}$ filter (Fisher Scientific), washed and then passed over a $40-\mu \mathrm{m}$ filter (Fisher Scientific). The cells were then incubated with anti-mouse Cd11b-coated microbeads (Miltenyi Biotech, Auburn, CA, USA) and then washed. The bead-cell pellet was resuspended in PBS/ $0.5 \% \mathrm{BSA} / 2 \mathrm{mM}$ EDTA and passed over a magnetic MACS Cell Separation column (Miltenyi Biotech) to separate Cd11b-positive cells (that is, cells that bound the beads-microglia/mononuclear phagocytes) from unbound Cd11b-negative cells $\left(\mathrm{CD} 11 \mathrm{~b}^{-}\right)$. Flow-through was collected and the column was rinsed $3 \times$ with PBS/ $\mathrm{BSA} / \mathrm{EDTA}$. CD11b-positive cells $\left(\mathrm{CD} 11 \mathrm{~b}^{+}\right)$were eluted by removing the column from the magnetic holder and pushing PBS/BSA/EDTA through the column with a plunger. Cells were centrifuged and the pellets were resuspended in RPMI 1640 (Invitrogen) and used for experiments. Microglia isolated in this manner are more than $96 \%$ pure $^{4}$

Isolation of monocytes from WT and knockout mice. Monocytes were isolated by centrifugation over Ficoll 1077 as described for human monocytes ${ }^{38,39}$. This step removes neutrophils and red blood cells. The remaining cells include monocytes and lymphocytes. This was followed by adhesion to tissue culture plates for $1 \mathrm{~h}$. The adherent cells ( $>90 \%$ monocytes) were then detached by brief incubation with $5 \mathrm{mM}$ EDTA in PBS as described ${ }^{38}$.

Quantitative real-time PCR. Total RNA from each sample of cells $\left(7.5-15 \times 10^{5}\right.$ cells) was isolated using the RNeasy Plus mini kit (Qiagen) according to the manufacturer's instructions and reverse transcribed using Multiscribe reverse transcriptase (Applied Biosystems, Foster City, CA, USA). Dilutions of each cDNA prep were used to assess $\beta 2$-microglobulin RNA levels, and samples were then adjusted to give equivalent levels of $\beta 2$-microglobulin per well in subsequent qPCR reactions for other genes. The qPCR was performed with the MX4000 unit (Agilent Technologies, Santa Clara, CA, USA) using SYBR Green to detect the amplification products as described ${ }^{1,5}$. The following cycles were performed: initial denaturation cycle $95^{\circ} \mathrm{C}$ for $10 \mathrm{~min}$, followed by 40 amplification cycles of $95^{\circ} \mathrm{C}$ for $15 \mathrm{~s}$ and $60{ }^{\circ} \mathrm{C}$ for $1 \mathrm{~min}$, and ending with one cycle at $25^{\circ} \mathrm{C}$ for $15 \mathrm{~s}$. Relative quantification of mRNA expression was calculated by the comparative cycle method described by the manufacturer (Agilent Technologies).

For Scara1 qPCR, a standard curve for Scaral was prepared from a plasmid (Open Biosystems, Huntsville, AL, USA) containing full-length mouse Scara1, and then whole-brain RNA was isolated from triplicate samples using a Qiagen RNA easy Mini kit and quantified using a Thermo-Fisher Nanodrop (Waltham, MA). RNA of $1 \mu \mathrm{g}$ was reverse transcribed into cDNA using Invitrogen's First Strand Synthesis kit (Carlsbad, CA, USA), and qPCR was performed in a Roche 480 Lightcycler qPCR machine (Indianapolis, IN, USA) in triplicates for Scara1 (Forward primer: $5^{\prime}$-TGAACGAGAGGATGCTGACTG-3', Reverse primer: $5^{\prime}$-GGAGGGGCCATTTTTAGTGC-3') and compared with a standard curve of mouse Scaral cDNA.

A $\boldsymbol{\beta}$ ELISA. For measurement of $A \beta$, we used commercially available ELISA kits (Invitrogen). Hemispheres of WT, PS1-APP and PS1-APP-Scara ${ }^{+1-}$ mice were homogenized in buffer containing $0.02 \mathrm{M}$ guanidine and ELISA for $\mathrm{A} \beta$ performed according to the manufacturer's instructions.

In vitro uptake of $\mathbf{A} \boldsymbol{\beta}$ by $\mathbf{N} \mathbf{9}$ cells. N9 cells cultured in six-well plates were incubated overnight with various concentrations of Protollin. The cells were then incubated with PBS containing $1 \% \mathrm{BSA}$ and $500 \mathrm{ng} \mathrm{ml}^{-1} \mathrm{~A} \beta 1-42$ labelled with Alexa 488 (Anaspec, CA) for up to $4 \mathrm{~h}$, then washed $3 \times$ in PBS and lifted from the plate with CellStripper (Mediatech). Cells were then fixed by the addition of $2 \%$ paraformaldehyde. Cell-associated fluorescence intensity was measured using a FACScalibur (BD Biosciences) flow cytometer.

Uptake of $A \beta$ from brain sections and immunohistochemistry. Brains from transgenic PS1-APP were collected and frozen unfixed in liquid nitrogen vapour and stored at $-80^{\circ} \mathrm{C}$, and $10-14-\mu \mathrm{m}$ frozen sections were cut. N9 cells or WT microglia isolated from adult mice were then added in the presence of Protollin and incubated at $37^{\circ} \mathrm{C}$ for $24 \mathrm{~h}$. Unbound cells were then rinsed in PBS, and the sections were fixed in $4 \%$ paraformaldehyde. To stain for $A \beta$, sections were blocked in $\mathrm{PBS} / 0.3 \%$ Triton $\mathrm{X}-100 / 2 \%$ goat serum for $30 \mathrm{~min}$, then incubated overnight at $25^{\circ} \mathrm{C}$ with rabbit anti-pan $\mathrm{A} \beta$ antibody (Biosource, Camarillo, CA, USA) or control antibody, at $2 \mu \mathrm{g} \mathrm{ml}^{-1}$ in PBS containing $0.3 \%$ Triton X-100 and $2 \%$ goat serum. The slides were then processed using the Vectastain Elite ABC Kit Rabbit IgG (Vector Laboratories, Burlingame, CA, USA) according to the manufacturer's instructions. Vector NovaRed substrate kit for peroxidase (Vector Laboratories) was used to develop target-bound peroxidase for detection of $A \beta$ in brain sections. Slides were counterstained with hematoxylin, mounted with VectaMount (Vector Laboratories), visualized by brightfield microscopy and digitally photographed. Alternatively, secondary anti rabbit IgG antibodies labelled with Alexa488 were used, and the slides were counterstained with 4',6-diamidino-2-phenylindole, mounted using Vectashield (Vector Laboratories) and digitally photographed. For quantitation of the percent surface area stained with Anti-A $\beta$, five equally spaced sections were used per brain.

Western blot of Scara1. Cell lysates of $2 \mu$ g were loaded onto a $10 \%$ SDS polyacrylamide gel (Invitrogen) transferred via iBlot (Invitrogen) onto polyvinylidene difluoride membrane, blocked for $1 \mathrm{~h}$ with $5 \%$ milk, then incubated with primary goat anti-mouse Scara1 antibodies (R\&D Systems) followed by anti-goat horseradish peroxidase-labelled secondary antibody. The blots were then exposed to Hyperfilm (Amersham), developed and band intensity-quantified by densitometry with image J software.

Statistical analysis. Statistical analysis was performed using one-way analysis of variance with the Tukey's test provided in the 'Microcal Origin 8' graphics and statistics software. $P$-values $<0.05$ where considered significant.

\section{References}

1. El Khoury, J. et al. Ccr2 deficiency impairs microglial accumulation and accelerates progression of Alzheimer-like disease. Nat. Med. 13, 432-438 (2007).

2. Hawkes, C. A. \& McLaurin, J. Selective targeting of perivascular macrophages for clearance of beta-amyloid in cerebral amyloid angiopathy. Proc. Natl Acad. Sci. USA 106, 1261-1266 (2009).

3. Town, T. et al. Blocking TGF-beta-Smad $2 / 3$ innate immune signalling mitigates Alzheimer-like pathology. Nat. Med. 14, 681-687 (2008).

4. Hickman, S. E., Allison, E. K. \& El Khoury, J. Microglial dysfunction and defective beta-amyloid clearance pathways in aging Alzheimer's disease mice. J. Neurosci. 28, 8354-8360 (2008).

5. El Khoury, J. B. et al. CD36 mediates the innate host response to beta-amyloid. J. Exp. Med. 197, 1657-1666 (2003).

6. Lue, L. F. et al. Soluble amyloid beta peptide concentration as a predictor of synaptic change in Alzheimer's disease. Am. J. Pathol. 155, 853-862 (1999).

7. McLean, C. A. et al. Soluble pool of Abeta amyloid as a determinant of severity of neurodegeneration in Alzheimer's disease. Ann. Neurol. 46, 860-866 (1999).

8. Wang, J., Dickson, D. W., Trojanowski, J. Q. \& Lee, V. M. The levels of soluble versus insoluble brain Abeta distinguish Alzheimer's disease from normal and pathologic aging. Exp. Neurol. 158, 328-337 (1999).

9. Haass, C. \& Selkoe, D. J. Soluble protein oligomers in neurodegeneration lessons from the Alzheimer's amyloid beta-peptide. Nat. Rev. Mol. Cell. Biol. 8, 101-112 (2007).

10. Sakono, M. \& Zako, T. Amyloid oligomers: formation and toxicity of Abeta oligomers. FEBS J. 277, 1348-1358 (2010).

11. Lesne, S. et al. A specific amyloid-beta protein assembly in the brain impairs memory. Nature 440, 352-357 (2006).

12. Walsh, D. M. et al. Naturally secreted oligomers of amyloid beta protein potently inhibit hippocampal long-term potentiation in vivo. Nature $\mathbf{4 1 6}$, 535-539 (2002).

13. Nimmerjahn, A., Kirchhoff, F. \& Helmchen, F. Resting microglial cells are highly dynamic surveillants of brain parenchyma in vivo. Science 308, 1314-1318 (2005).

14. El Khoury, J. et al. Scavenger receptor-mediated adhesion of microglia to betaamyloid fibrils. Nature 382, 716-719 (1996).

15. Coraci, I. S. et al. CD36, a class B scavenger receptor, is expressed on microglia in Alzheimer's disease brains and can mediate production of reactive oxygen species in response to beta-amyloid fibrils. Am. J. Pathol. 160, 101-112 (2002).

16. Yan, S. D. et al. RAGE and amyloid-beta peptide neurotoxicity in Alzheimer's disease. Nature 382, 685-691 (1996).

17. El Khoury Joseph, Hickman Suzanne E. Mechanisms of Amyloid B Clearance in Alzheimer's Disease (Nova Biomedical Books, New York, 2009).

18. Moffat, J. et al. A lentiviral RNAi library for human and mouse genes applied to an arrayed viral high-content screen. Cell 124, 1283-1298 (2006). 
19. Means, T. K. et al. Evolutionarily conserved recognition and innate immunity to fungal pathogens by the scavenger receptors SCARF1 and CD36. J. Exp. Med. 206, 637-653 (2009).

20. Wilkinson, K., Boyd, J. D., Glicksman, M., Moore, K. J. \& El Khoury, J. A highcontent drug screen identifies ursolic acid as an inhibitor of amyloid-\{beta\} interactions with its receptor CD36. J. Biol. Chem. 286, 34914-34922 (2011).

21. Stewart, C. R. et al. CD36 ligands promote sterile inflammation through assembly of a Toll-like receptor 4 and 6 heterodimer. Nat. Immunol. 11, 155-161 (2010).

22. Maxeiner, H. et al. Complementary roles for scavenger receptor A and CD36 of human monocyte-derived macrophages in adhesion to surfaces coated with oxidized low-density lipoproteins and in secretion of H2O2. J. Exp. Med. 188, 2257-2265 (1998)

23. Fries, L. F. et al. Safety and immunogenicity of a proteosome-Shigella flexneri 2a lipopolysaccharide vaccine administered intranasally to healthy adults. Infect. Immun. 69, 4545-4553 (2001).

24. Frenkel, D., Maron, R., Burt, D. S. \& Weiner, H. L. Nasal vaccination with a proteosome-based adjuvant and glatiramer acetate clears beta-amyloid in a mouse model of Alzheimer disease. J. Clin. Invest. 115, 2423-2433 (2005).

25. Frenkel, D. et al. A nasal proteosome adjuvant activates microglia and prevents amyloid deposition. Ann. Neurol. 63, 591-601 (2008).

26. Wyss-Coray, T. et al. Adult mouse astrocytes degrade amyloid-beta in vitro and in situ. Nat. Med. 9, 453-457 (2003).

27. El Khoury, J. Neurodegeneration and the neuroimmune system. Nat. Med. 16, 1369-1370.

28. Grathwohl, S. A. et al. Formation and maintenance of Alzheimer's disease betaamyloid plaques in the absence of microglia. Nat. Neurosci. 12, 1361-1363 (2009).

29. Bruban, J. et al. CCR2/CCL2-mediated inflammation protects photoreceptor cells from amyloid-beta-induced apoptosis. Neurobiol. Dis. 42, 55-72 (2011).

30. Reed-Geaghan, E. G., Reed, Q. W., Cramer, P. E. \& Landreth, G. E. Deletion of CD14 attenuates Alzheimer's disease pathology by influencing the brain's inflammatory milieu. J. Neurosci. 30, 15369-15373 (2010).

31. Song, M. et al. TLR4 mutation reduces microglial activation, increases Abeta deposits and exacerbates cognitive deficits in a mouse model of Alzheimer's disease. J. Neuroinflammation 8, 92 (2011).

32. Mildner, A. et al. Distinct and non-redundant roles of microglia and myeloid subsets in mouse models of Alzheimer's disease. J. Neurosci. 31, 11159-11171 (2011).

33. Borchelt, D. R. et al. Accelerated amyloid deposition in the brains of transgenic mice coexpressing mutant presenilin 1 and amyloid precursor proteins. Neuron 19, 939-945 (1997).
34. Jankowsky, J. L. et al. Co-expression of multiple transgenes in mouse CNS: a comparison of strategies. Biomol. Eng. 17, 157-165 (2001).

35. Thomas, C. A. et al. Protection from lethal gram-positive infection by macrophage scavenger receptor-dependent phagocytosis. J. Exp. Med. 191, 147-156 (2000).

36. Mehta, J. L. et al. Deletion of LOX-1 reduces atherogenesis in LDLR knockout mice fed high cholesterol diet. Circ. Res. 100, 1634-1642 (2007).

37. Yonekura, H., Yamamoto, Y., Sakurai, S., Watanabe, T. \& Yamamoto, H. Roles of the receptor for advanced glycation endproducts in diabetes-induced vascular injury. J. Pharmacol. Sci. 97, 305-311 (2005).

38. el Khoury, J. et al. Macrophages adhere to glucose-modified basement membrane collagen IV via their scavenger receptors. J. Biol. Chem. 269, 10197-10200 (1994).

39. Hickman, S. E., el Khoury, J., Greenberg, S., Schieren, I. \& Silverstein, S. C. P2Z adenosine triphosphate receptor activity in cultured human monocyte-derived macrophages. Blood 84, 2452-2456 (1994).

\section{Acknowledgements}

This work was supported by a grant from the HFSP and ISF and an NIRG grant from the Alzheimer's Association to D.F., by grant AG043975 to H.L.W. and by NIH grants NS059005, AG032349 and AI082660 and a grant from the Dana Foundation Neuroimmunology Program to J.E.K.

\section{Author contributions}

D.F. and K.W. designed, performed and analysed experiments. L.Z., S.E.H., T.K.M., L.P., D.F. and N.D.K. performed and analysed experiments. H.L.W. designed experiments and edited manuscript. J.E.K. designed, supervised and analysed experiments, and wrote the manuscript.

\section{Additional information}

Supplementary Information accompanies this paper at http://www.nature.com/ naturecommunications

Competing financial interests: The authors declare no competing financial interests.

Reprints and permission information is available online at http://npg.nature.com/ reprintsandpermissions/

How to cite this article: Frenkel, D. et al. Scara1 deficiency impairs clearance of soluble amyloid- $\beta$ by mononuclear phagocytes and accelerates Alzheimer's-like disease progression. Nat. Commun. 4:2030 doi: 10.1038/ncomms3030 (2013). 